\title{
Layer-by-Layer Assembled Bacterial Cellulose/Graphene Oxide Hydrogels with Extremely Enhanced Mechanical Properties
}

\author{
Honglin Luo ${ }^{1,2} \cdot$ Jiaojiao Dong ${ }^{1}$ Fanglian Yao ${ }^{3}$ Zhiwei Yang ${ }^{1} \cdot \mathrm{Wei} \mathrm{Li}^{1} \cdot \mathrm{Jie} \mathrm{Wang}^{1} \cdot \mathrm{Xinhua} \mathrm{Xu}^{2}$. \\ Jian $\mathrm{Hu}^{1} \cdot$ Yizao Wan ${ }^{1,2}$
}

Received: 30 December 2017 / Accepted: 27 February 2018/Published online: 27 March 2018

(C) The Author(s) 2018

\section{Highlights}

- A modified in situ static culture method (layer-by-layer assembly, LBLA) was developed.

- The LBLA method ensures uniform distribution of graphene oxide (GO) in bacterial cellulose (BC) and makes very thick BC/GO hydrogels with homogeneous structures.

- The BC/GO hydrogels show greatly enhanced mechanical properties over bare BC.

\begin{abstract}
Uniform dispersion of two-dimensional (2D) graphene materials in polymer matrices remains challenging. In this work, a novel layer-by-layer assembly strategy was developed to prepare a sophisticated nanostructure with highly dispersed 2D graphene oxide in a three-dimensional matrix consisting of onedimensional bacterial cellulose (BC) nanofibers. This method is a breakthrough, with respect to the conventional static culture method for BC that involves multiple in situ layer-by-layer assembly steps at the interface between previously grown $\mathrm{BC}$ and the culture medium. In the as-prepared BC/GO nanocomposites, the GO nanosheets are mechanically bundled and chemically bonded with $\mathrm{BC}$ nanofibers via hydrogen bonding,
\end{abstract}

Jian $\mathrm{Hu}$

jhu11s@alum.imr.ac.cn

$\triangle$ Yizao Wan

yzwan@tju.edu.cn

1 School of Materials Science and Engineering, East China Jiaotong University, Nanchang 330013, People's Republic of China

2 School of Materials Science and Engineering, Tianjin Key Laboratory of Composite and Functional Materials, Key Laboratory of Advanced Ceramics and Machining Technology, Ministry of Education, Tianjin University, Tianjin 300072, People's Republic of China

3 School of Chemical Engineering, Tianjin University, Tianjin 300072, People's Republic of China
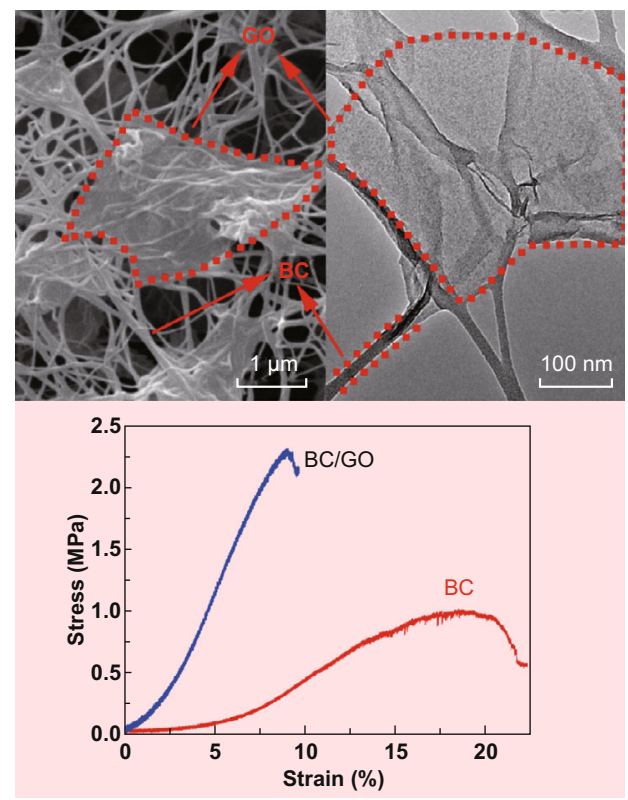

forming an intriguing nanostructure. The sophisticated nanostructure of the $\mathrm{BC} / \mathrm{GO}$ leads to greatly enhanced mechanical properties compared to those of bare $\mathrm{BC}$. This strategy is versatile, facile, scalable, and can be promising for the development of high-performance BC-based nanocomposite hydrogels.

Keywords Bacterial cellulose - Nanocomposite · Graphene oxide $\cdot$ Biosynthesis $\cdot$ Nanofiber $\cdot$ Hydrogels 


\section{Introduction}

Nano-carbon materials, such as one-dimensional (1D) carbon nanotube (CNT) and two-dimensional (2D) graphene (GE) and graphene oxide (GO), are believed to be promising candidate materials for tissue engineering and regenerative medicine applications owing to their large specific surface area, high porosity, and excellent mechanical properties [1-7]. Among these carbonaceous nanomaterials, GO is considered a promising material for biological applications owing to its excellent biocompatibility, better dispersibility in water than GE, and abundant surface functional groups [8-12]. Furthermore, GO can support and accelerate adhesion, proliferation, and differentiation of various mammalian cells [12-15].

Despite its solubility in water, GO tends to aggregate in physiological environments because of nonspecific binding to proteins [16], which will inevitably lower its reinforcement effectiveness when used as nanofillers in GO-based nanocomposites. To make full use of its good dispersion in water, GO has been used to reinforce a natural polymer, bacterial cellulose (BC), to form $\mathrm{BC} / \mathrm{GO}$ nanocomposites. For instance, Feng et al. [17] prepared the BC/GO nanocomposite by mechanically mixing $\mathrm{BC}$ fragments with a GO aqueous dispersion. The obtained nanocomposites show a tensile strength of $242 \mathrm{MPa}$ in the dry state (5 wt $\%$ $\mathrm{GO}$ ), which is an improvement of $22 \%$ compared to that of bare BC (198 MPa). Liu et al. [18] prepared a BC/GO nanocomposite (in the dry state) with a tensile strength of $18.48 \mathrm{MPa}$ by a one-step cross-linking method. In both cases, however, the obtained nanocomposites broke the intrinsically three-dimensional (3D) structure of the $\mathrm{BC}$, which is the most precious feature distinguishing it from other natural polymers. Incorporation of GE or CNTs into the inner core of a 3D BC network, by filtration or postimmersion in a solution of GE or CNTs, is challenging because of the lack of large pores $(>20 \mu \mathrm{m})$ in pristine BC [19].

In order to retain the advantageous $3 \mathrm{D}$ structure of $\mathrm{BC}$, Yoon et al. [20] reported a post-processing immersion method to fabricate BC-CNT nanocomposites by immersing $\mathrm{BC}$ pellicles in CNT solutions. However, this method cannot work when the $\mathrm{BC}$ pellicles are thick. More importantly, in the case of GO, the post-processing immersion method might not be feasible for the preparation of $\mathrm{BC} / \mathrm{GO}$ nanocomposites, since $\mathrm{GO}$ is much larger than $\mathrm{CNT}$ and thus cannot enter the inner structure of BC pellicles. In our previous studies, a one-pot in situ biosynthesis approach was developed, and a $\mathrm{BC} / \mathrm{GO}$ nanocomposite with homogeneous $\mathrm{GO}$ nanosheets in a $\mathrm{BC}$ matrix was successfully fabricated [21]. The $\mathrm{BC} / \mathrm{GO}$ nanocomposite (in a dry state) showed high mechanical properties and improved electrical conductivity, compared to those of the pristine BC. Unfortunately, neither post-processing immersion nor in situ biosynthesis could enable GO to penetrate the internal area of the $\mathrm{BC}$ network when the $\mathrm{BC} /$ GO hydrogel was thicker than $2 \mathrm{~mm}$. Therefore, a great deal of effort is required to improve the uniform dispersion of $\mathrm{GO}$ in a $3 \mathrm{D}$ integrated $\mathrm{BC}$ matrix, particularly when thick $\mathrm{BC} / \mathrm{GO}$ products are required.

Herein, we report a novel in situ layer-by-layer assembly (LBLA) method for fabricating thick $(\geq 5 \mathrm{~mm}$ ) BC/ GO nanocomposite hydrogels with highly dispersed GO nanosheets bundled by 3D interconnected BC nanofibers. The sophisticated porous structures and improved mechanical properties of the as-prepared $\mathrm{BC} / \mathrm{GO}$ nanocomposites were investigated carefully.

\section{Experimental}

\subsection{Materials and Methods}

Yeast extract, tryptone, disodium phosphate $\left(\mathrm{Na}_{2} \mathrm{HPO}_{4}\right)$, and acetic acid were used as-received for $\mathrm{BC}$ production. A commercially available aqueous dispersion of GO with a concentration of $0.5 \mathrm{mg} \mathrm{mL}^{-1}$ was purchased from Nanjing XFNANO Materials Technology Co. Ltd., China. The bacterial strain, Komagataeibacter xylinus X-2, was kindly provided by Tianjin University of Science and Technology, Tianjin, China.

\subsection{Preparation of $\mathrm{BC}, \mathrm{c}-\mathrm{BC} / \mathrm{GO}$, and $\mathrm{BC} / \mathrm{GO}$}

Prior to inoculation, the culture medium $(\mathrm{pH}=4.5)$ of $\mathrm{BC}$, composed of $2.5 \%(\mathrm{w} / \mathrm{v})$ glucose, $0.75 \%(\mathrm{w} / \mathrm{v})$ yeast extract, $1 \%(\mathrm{w} / \mathrm{v})$ tryptone, and $1 \%(\mathrm{w} / \mathrm{v}) \mathrm{Na}_{2} \mathrm{HPO}_{4}$, was sterilized at $121{ }^{\circ} \mathrm{C}$ for $30 \mathrm{~min}$. This recipe has been reported in our previous work $[22,23]$. To prepare BC/GO nanocomposite hydrogels, the GO suspension was added to the above-mentioned culture medium of $\mathrm{BC}$ under intense stirring for $60 \mathrm{~min}$. A pure $\mathrm{BC}$ membrane $(\approx 3 \mathrm{~mm}$ in thickness and denoted as $\mathrm{BC}_{0}$ hereinafter), prepared by a conventional static culture method, was placed in a culture dish. Afterward, the GO-dispersed culture medium was sprayed onto the surface of the as-obtained $\mathrm{BC}_{0}$ membrane, on which new $\mathrm{BC}$ grew at the interface of the $\mathrm{BC}_{0}$ membrane and the GO-dispersed culture medium, leading to a $\mathrm{BC} / \mathrm{GO}$ film. When the medium was consumed, additional GO-dispersed culture medium was sprayed onto the surface of the newly prepared BC/GO film, and the second layer of 
the $\mathrm{BC} / \mathrm{GO}$ film was produced. The process continued until the desired hydrogel thickness was reached.

The GO content in BC/GO nanocomposites (with respect to hydrated $\mathrm{BC}$ ) was adjusted by the volume ratio of $\mathrm{BC}$ culture medium to GO dispersion (Table 1). Three $\mathrm{BC} / \mathrm{GO}$ nanocomposite hydrogels, namely $\mathrm{BC} / \mathrm{GO}-1, \mathrm{BC} /$ GO-2, and BC/GO-3, were prepared, which contained 0.11, 0.16 , and $0.22 \%$ of GO by mass (wt $\%$ ), respectively (Table 1). The GO contents were obtained by measuring the weight differences of $\mathrm{BC}$ and $\mathrm{BC} / \mathrm{GO}$ hydrogels with identical volumes, since GO addition does not affect the production of BC. For comparison, a BC/GO hydrogel with a thickness of $5 \mathrm{~mm}$ was prepared by conventional in situ biosynthesis [21] at a volume ratio of $V_{\text {culture medium }} /$ $V_{\mathrm{GO}}$ dispersion $=3: 1$, which was designated as c-BC/GO-2. Additionally, pure $\mathrm{BC}$ hydrogels were prepared following the same procedure using a GO-free culture medium. The harvested hydrogels were purified using the previously reported procedure [21], providing $\mathrm{BC} / \mathrm{GO}$ and $\mathrm{BC}$ hydrogels for mechanical testing. For the other characterization methods, the hydrogels were freeze-dried to produce aerogels.

\subsection{Characterization Methods}

The photographs were taken with a digital camera. The morphologies of the $\mathrm{BC}$ and $\mathrm{BC} / \mathrm{GO}$ aerogels were observed using a field emission scanning electron microscope (FE-SEM, Nano 430, FEI, USA) and transmission electron microscope (TEM, JEOL, Japan) operating at $200 \mathrm{kV}$. Fourier transform infrared spectroscopy (FTIR) was performed using a Bruker Optik GmbH spectrometer. $\mathrm{X}$-ray photoelectron spectroscopy was performed with a PHI Quantera X-ray photoelectron spectroscope (XPS, ULVAC-PHI, Inc., Japan). The crystallographic structure was analyzed on a D8 Advance X-ray diffractometer (XRD) using $\mathrm{Cu} \mathrm{K} \alpha$ radiation $(\lambda=0.154 \mathrm{~nm})$ at the scanning range of $10^{\circ}-50^{\circ}$ at a scan speed of $0.02^{\circ} \mathrm{s}^{-1}$; the data were obtained using the MDI/JADE6 software package attached to the Rigaku XRD instrument. The crystallinity index (CI) was calculated by Segal's method using Eq. 1 [24]:

$\mathrm{CI}=\left(I_{200}-I_{a m}\right) / I_{200}$

where $I_{200}$ is the intensity at a $2 \theta$ value of $22^{\circ}$ and $I_{a m}$ is the intensity of the baseline at a $2 \theta$ value of $18^{\circ}$.
Thermogravimetric analysis (TGA) was conducted using a TGA instrument (STA449F3) with a heating rate of $10{ }^{\circ} \mathrm{C} \mathrm{min}{ }^{-1}$ from room temperature to $800{ }^{\circ} \mathrm{C}$. The Brunauer-Emmett-Teller (BET) surface area, pore size, and volume were measured by the nitrogen adsorption method using a surface area analyzer (NOVA 2200e).

Static tensile tests of $\mathrm{BC}$ and $\mathrm{BC} / \mathrm{GO}$ hydrogels (dimensions: $50 \times 10 \times 2 \mathrm{~mm}^{3}$ ) were conducted using a micro-electromagnetic fatigue testing machine (MUF1050, Tianjin Care Measure \& Control Co., Ltd., Tianjin, China) at a strain rate of $0.1 \mathrm{~mm} \mathrm{~s}^{-1}$. At least five specimens were tested for each sample, and the averages and standard deviations were reported.

\section{Results and Discussion}

The typical fabrication process for the BC/GO nanocomposite hydrogel is schematically illustrated in Fig. 1. Prior to LBLA, a base membrane (a $\mathrm{BC}_{0}$ film) was prepared by a conventional static culture method. This $\mathrm{BC}_{0}$ base membrane was placed in a container (e.g., a disk). The LBLA method includes numerous cycles, each of which involves two consecutive steps. The first step is to spray the GOcontaining culture medium (aqueous suspension, $\approx 0.5 \mathrm{~mL}$ ) as droplets onto the surface of the $\mathrm{BC}_{0}$ base membrane such that the LBLA process (namely biosynthesis, the second step) initiates, resulting in the growth of a BC/GO film ( $\approx 0.2-0.4 \mathrm{~mm}$ in thickness $)$ on the surface of the base membrane with the help of sterilized oxygen. In the second cycle, the resultant BC/GO film serves as a new base membrane on which the second $\mathrm{BC} / \mathrm{GO}$ film (the same thickness as the first $\mathrm{BC} / \mathrm{GO}$ film) grows via biosynthesis. These cycles repeat until a predefined hydrogel thickness $(\approx 2 \mathrm{~mm}$ for tensile testing and $\approx 5 \mathrm{~mm}$ for morphology analysis) is reached. The last step is the removal of the base membrane and purification of the hydrogel, leaving a freestanding $\mathrm{BC} / \mathrm{GO}$ nanocomposite hydrogel.

Figure 2 shows the photographs of the $\mathrm{c}-\mathrm{BC} / \mathrm{GO}-2$ and $\mathrm{BC} / \mathrm{GO}-2$ hydrogels and the morphology of the $\mathrm{BC}$ and $\mathrm{BC} / \mathrm{GO}$ aerogels. As shown in Fig. 2a, the digital photograph of c-BC/GO clearly demonstrates that there is a GO-free thin layer on top of the c-BC/GO sample (Fig. $2 \mathrm{a}_{1}$ ), which suggests that the maximum thickness of a
Table 1 GO content in various $\mathrm{BC} / \mathrm{GO}$ nanocomposite hydrogels prepared in this work

\begin{tabular}{lll}
\hline Sample No. & $V_{\text {culture medium }} / V_{\mathrm{GO}}$ dispersion & GO content with respect to BC (wt\%) \\
\hline BC/GO-1 & $5: 1$ & 0.11 \\
BC/GO-2 & $3: 1$ & 0.16 \\
BC/GO-3 & $1: 1$ & 0.22 \\
c-BC/GO-2 & $3: 1$ & - \\
\hline
\end{tabular}



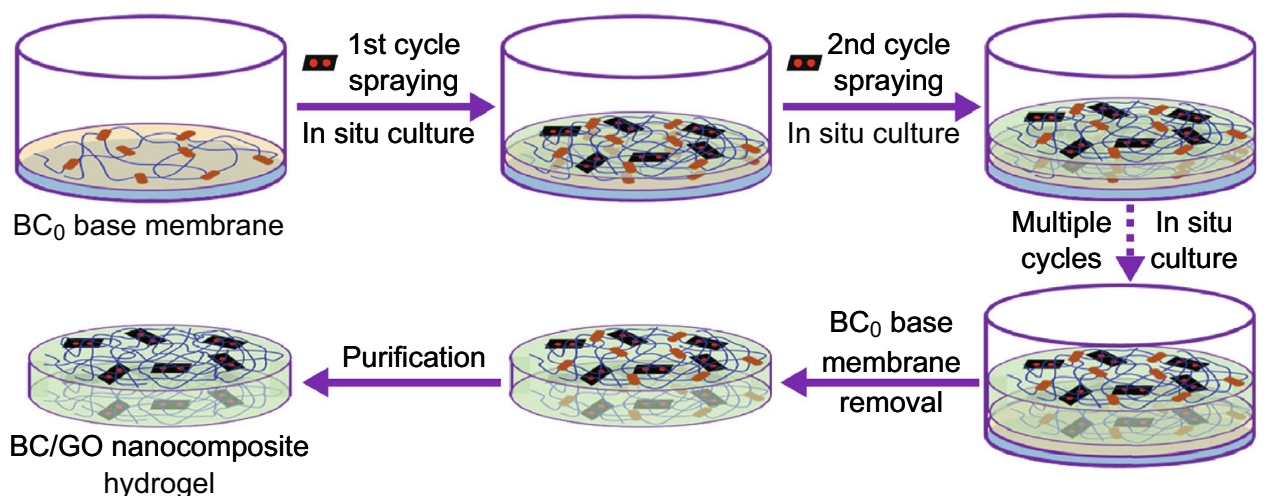

Bacteria $\sim \mathrm{BC}$ fiber $\boldsymbol{\varpi G O}$ nanosheet • Oxygen containing functional group

Fig. 1 Schematic illustration of the typical fabrication process of BC/GO nanocomposite hydrogel. The mixture of GO suspension and culture medium of $\mathrm{BC}$ was sprayed onto the $\mathrm{BC}_{0}$ base film (in the first cycle) or the newly grown $\mathrm{BC} / \mathrm{GO}$ (in the subsequent cycles) followed by in situ culture
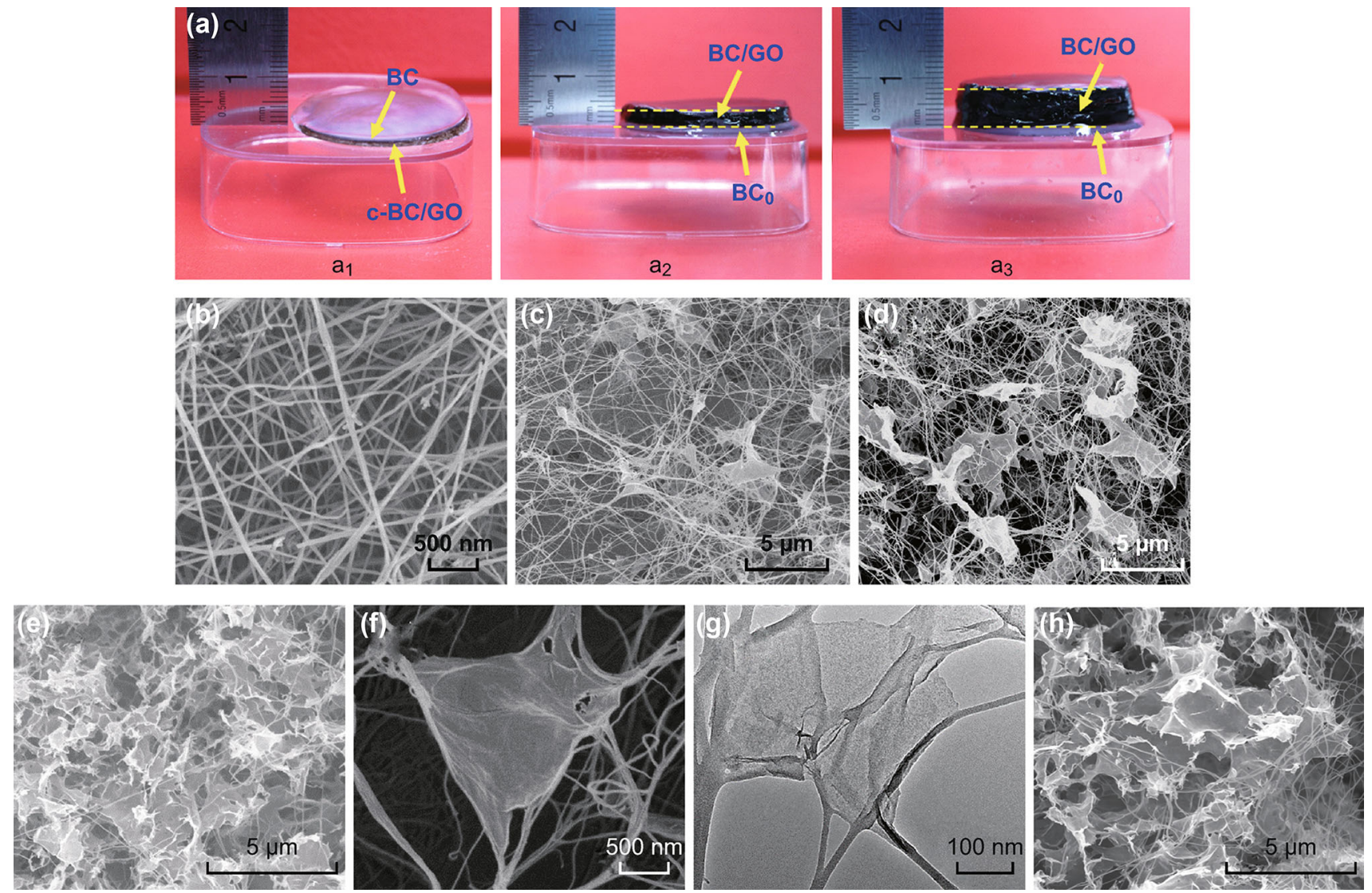

Fig. 2 a Digital photographs of c-BC/GO and BC/GO hydrogels. SEM images taken from the surface layers of $\mathbf{b}$ BC, $\mathbf{c}$ BC/GO-1, d BC/GO-2, and $\mathbf{e}$ BC/GO-3. f Magnified SEM and $\mathbf{g}$ TEM images of the "leaf and vein" nanostructure of BC/GO-2. h A representative SEM image of BC/ GO-3 from central area indicating the uniform structure

c-BC/GO composite is $\approx 2 \mathrm{~mm}$ for conventional in situ biosynthesis. This is likely due to the gradual decrease in the GO content in the culture medium during the fermentation process; however, the exact mechanism is not yet fully understood. In contrast, a uniform black BC/GO-2 hydrogel is noted with a thickness of either $3 \mathrm{~mm}$ (Fig. $2 \mathrm{a}_{2}$ ) or even $7 \mathrm{~mm}$ (Fig. $2 \mathrm{a}_{3}$ ), which indicates that the LBLA strategy can produce BC/GO hydrogels with uniformly distributed GO. To further observe the distribution of GO in the BC matrix, SEM and TEM analyses were 

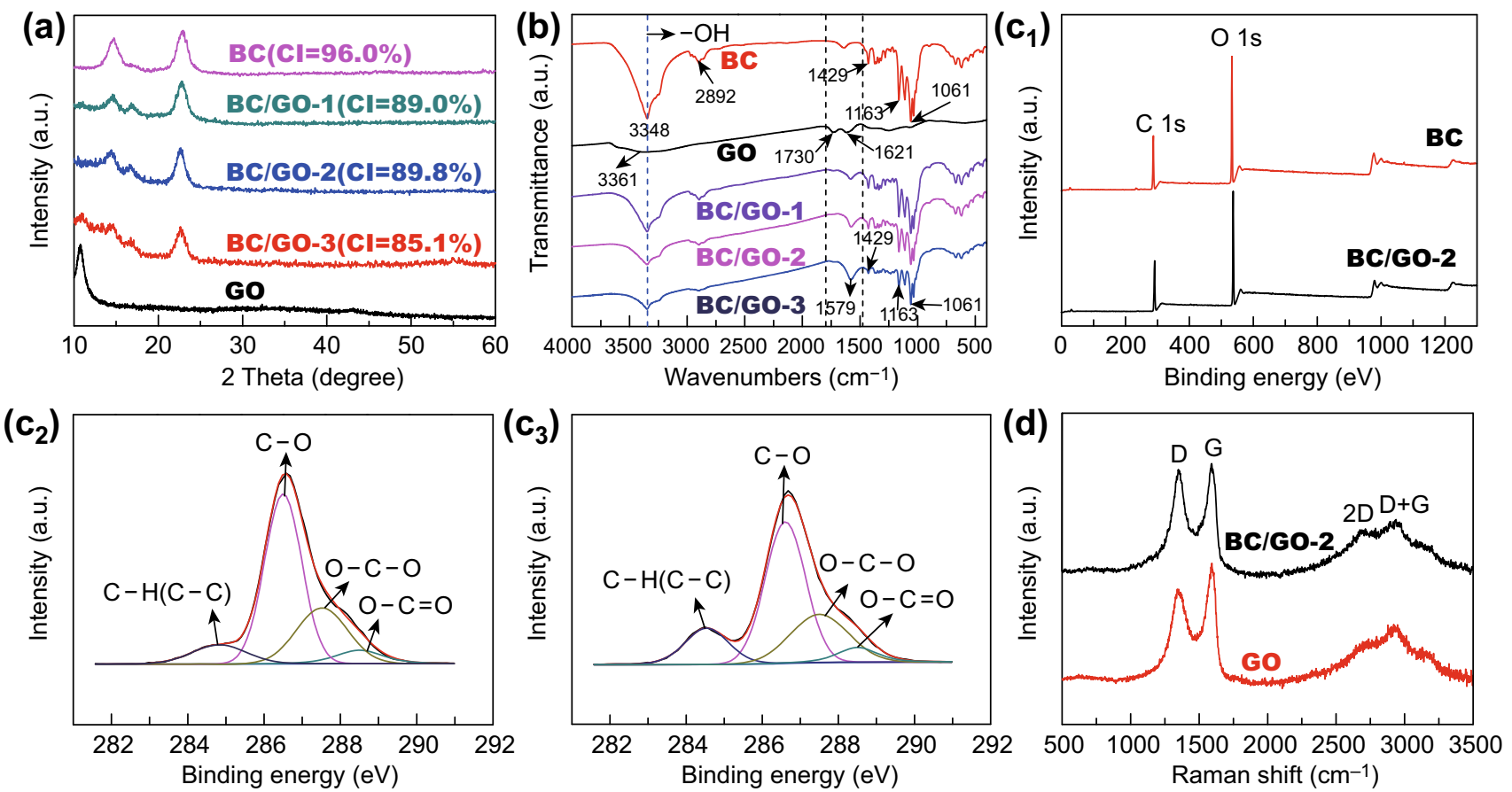

Fig. 3 a XRD patterns of $\mathrm{BC}_{0}, \mathrm{BC}$, and $\mathrm{BC} / \mathrm{GO}$ nanocomposites. b FTIR spectra of BC, GO, and BC/GO nanocomposites. c Wide-scan XPS spectra of $\mathrm{BC}$ and $\mathrm{BC} / \mathrm{GO}-2\left(\mathrm{c}_{1}\right)$, high-resolution $\mathrm{C}$ 1s spectra of $\mathrm{BC}\left(\mathrm{c}_{2}\right)$ and $\mathrm{BC} / \mathrm{GO}-2\left(\mathrm{c}_{3}\right)$. d Raman spectra of pristine GO and $\mathrm{BC} / \mathrm{GO}-2$ nanocomposite

conducted. Figure $2 \mathrm{~b}$ displays a typical $3 \mathrm{D}$ porous interconnected nanostructure of $\mathrm{BC}$, which is similar to that reported in our previous work [21, 25], indicating that the LBLA process does not affect the morphology of the resultant BC. As seen in Fig. 2c-e, GO is uniformly distributed in the $\mathrm{BC}$ network in $\mathrm{BC} / \mathrm{GO}-1, \mathrm{BC} / \mathrm{GO}-2$, and $\mathrm{BC} / \mathrm{GO}-3$. Figure $2 \mathrm{f}$ reveals a typical entangled structure. To further examine the morphology of BC/GO nanocomposites, TEM observation was performed that further confirmed the entangled structure (Fig. $2 \mathrm{~g}$ ). Figure $2 \mathrm{~h}$ shows a typical image of BC/GO-2 from the central part, which demonstrates the uniform distribution of GO in the core area of BC/GO-2. This finding further shows the great capability of the LBLA method in dispersing GO nanosheets throughout the BC matrix. It should be pointed out that higher than $0.22 \mathrm{wt} \%$ GO content will result in GO aggregation in $\mathrm{BC} / \mathrm{GO}$ aerogels owing to the ultra-thinness of GO nanosheets. Therefore, in this work, $0.22 \mathrm{wt} \%$ is the highest GO content observed for the $\mathrm{BC} / \mathrm{GO}$ nanocomposite hydrogels.

Figure 3a shows the XRD patterns of BC/GO nanocomposites, GO, and $\mathrm{BC}$. It is noted that the positions $\left(14.6^{\circ}, 16.8^{\circ}\right.$, and $\left.22.8^{\circ}\right)$ of three characteristic peaks, namely the (11̄0), (110), and (200) BC diffraction planes, do not change in the XRD patterns of the $\mathrm{BC} / \mathrm{GO}$ nanocomposites as compared to $\mathrm{BC}$, indicating that the crystalline structure (lattice parameters) of $\mathrm{BC}$ remains unchanged upon incorporation of GO in the culture medium of the BC. However, the crystallinity indices $(C I)$ of the three BC/GO samples are significantly lower than that of BC (Fig. 3a). This result indicates that the presence of $\mathrm{GO}$ in the $\mathrm{BC}$ culture medium has an effect on the formation process of $\mathrm{BC}$ crystals, which is generally ascribed to the disturbance by additives to the formation of $\mathrm{BC}$ nanofibrils [17, 26, 27]. The smaller $C I$ of $\mathrm{BC} / \mathrm{GO}-3$ versus those of $\mathrm{BC} / \mathrm{GO}-1$ and $\mathrm{BC} / \mathrm{GO}-2$ indicates that the $\mathrm{GO}$ content has an impact on CI. However, how the concentration of additives affects the formation process is unclear. It is interesting to note that there is no GO peak in the XRD patterns of $\mathrm{BC} / \mathrm{GO}-1$ and $\mathrm{BC} / \mathrm{GO}-2$, which may indicate even distribution of GO in the 3D BC matrix [25, 28-31], consistent with SEM observation. However, a weak peak is located at $\approx 11^{\circ}$ in the XRD pattern of BC/GO-3, which is the same as the intense peak in the XRD pattern of bare GO. The presence of this peak may suggest slight GO aggregation in $\mathrm{BC} / \mathrm{GO}-3$, although SEM cannot clearly show this trend.

Surface chemistry of the BC/GO nanocomposites was examined by FTIR analysis (Fig. 3b). In the spectrum of BC, characteristic peaks $(3348,2892,1429$, and $1061 \mathrm{~cm}^{-1}$ due to $-\mathrm{OH}$ bonds, asymmetric stretching vibration of $\mathrm{C}-\mathrm{H}$, asymmetric angular deformation of $\mathrm{C}-\mathrm{H}$ 

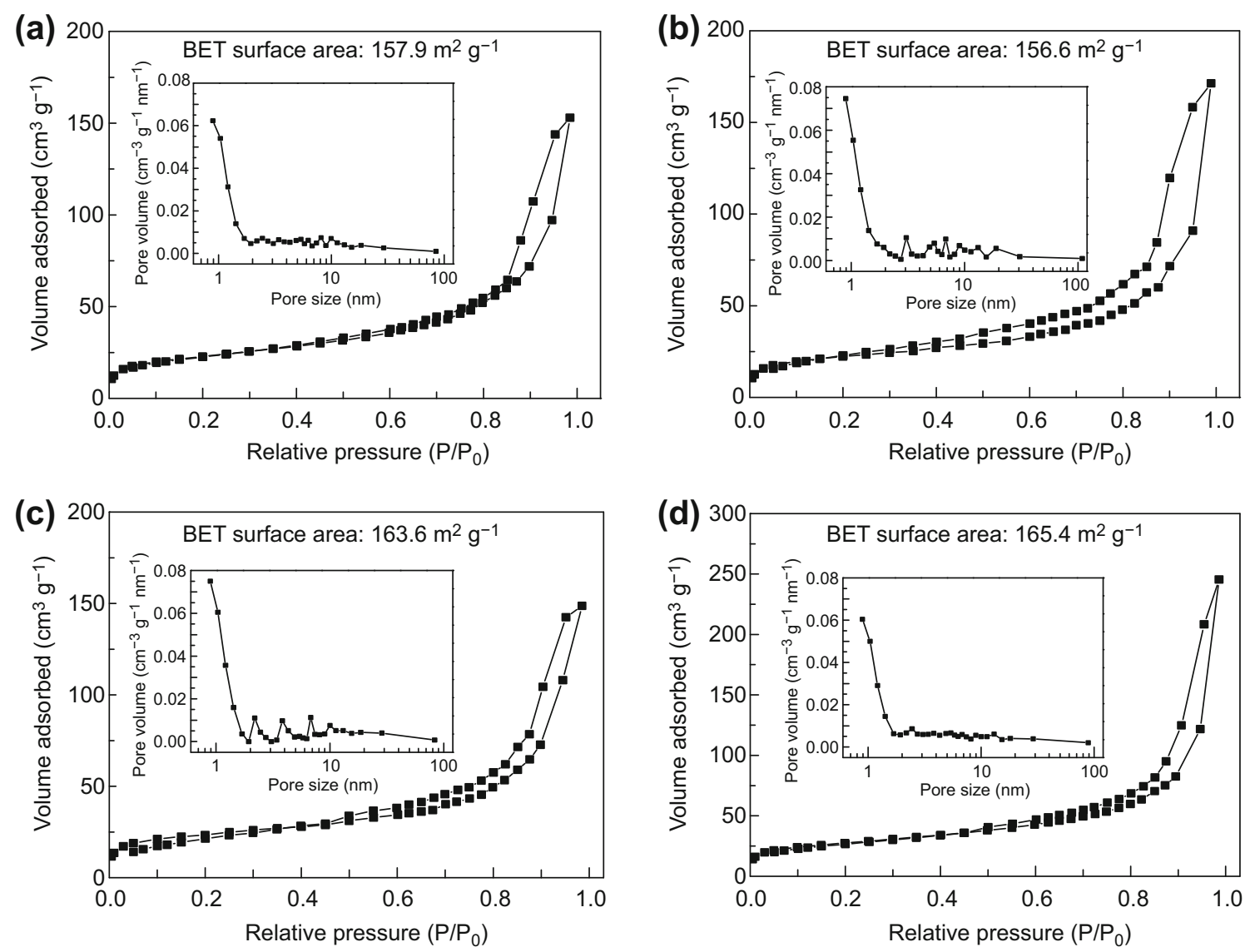

Fig. 4 The nitrogen adsorption/desorption curves and pore size distribution curves of a BC, b BC/GO-1, c BC/GO-2, and d BC/GO-3

bonds, and antisymmetric bridge stretching of $\mathrm{C}-\mathrm{O}-\mathrm{C}$, respectively [18, 32-34]) are observed. In the spectrum of GO, three peaks, centered at 3361, 1730, and $1621 \mathrm{~cm}^{-1}$, corresponding to the stretching vibrations of $\mathrm{O}-\mathrm{H}, \mathrm{C}=\mathrm{O}$, and $\mathrm{C}=\mathrm{C}$ bonds $[17,35]$, respectively, are observed. The major characteristic absorptions of $\mathrm{GO}$ and $\mathrm{BC}$ are also noted in the $\mathrm{BC} / \mathrm{GO}$ nanocomposites. It is noted that the peak intensity of the $-\mathrm{OH}$ group $\left(3348 \mathrm{~cm}^{-1}\right)$ decreases with increasing $\mathrm{GO}$ content in the $\mathrm{BC} / \mathrm{GO}$ nanocomposites as compared with that observed with pure BC. In addition, an intense peak at $1579 \mathrm{~cm}^{-1}$ is noted, and its intensity increases with the GO content. This may indicate a strong interaction (hydrogen bonding) between $\mathrm{BC}$ and $\mathrm{GO}$, which causes downshift of the $\mathrm{GO} \mathrm{C}=\mathrm{O}$ group band. The formation of hydrogen bonding is important for enhancing the mechanical properties of BC/GO composites [36, 37].

To further determine the surface chemistry of the BC/ GO nanocomposites, XPS analysis was carried out, and the results are presented in Fig. 3c. Both $\mathrm{BC}$ and $\mathrm{BC} / \mathrm{GO}-2$ show almost identical wide-scan spectra (Fig. $3 \mathrm{c}_{1}$ ). Furthermore, it seems that the $\mathrm{C} 1 s$ spectra of $\mathrm{BC}$ and $\mathrm{BC} / \mathrm{GO}-$ 2 are the same, both showing four peaks of $\mathrm{C}-\mathrm{C}$ or $\mathrm{C}-\mathrm{H}$
(284.6 eV), C-O (286.5 eV), O-C-O (287.5 eV), and $\mathrm{O}-$ $\mathrm{C}=\mathrm{O}(288.5 \mathrm{eV})$. However, a careful comparison reveals a difference in the sub-peak intensity of $\mathrm{C}-\mathrm{C}$ or $\mathrm{C}-\mathrm{H}$. As expected, the incorporation of $\mathrm{GO}$ improves the $\mathrm{C}-\mathrm{C}$ or $\mathrm{C}-$ $\mathrm{H}$ peak strength of $\mathrm{BC} / \mathrm{GO}-2$.

Figure $3 \mathrm{~d}$ presents the Raman spectra of $\mathrm{GO}$ and $\mathrm{BC} /$ GO-2. The two curves are similar, both showing a D band (1344 $\mathrm{cm}^{-1}$, which corresponds to the disordered structure of GO sheets) and a $\mathrm{G}$ band $\left(1595 \mathrm{~cm}^{-1}\right.$, which represents the first-order scattering of the $E_{2 \mathrm{~g}}$ vibrational mode) $[38,39]$. However, determination of the intensity ratio of $\mathrm{D}$ band to $\mathrm{G}$ band, $I_{\mathrm{D}} / I_{\mathrm{G}}$, reveals a significant difference between GO (0.86) and BC/GO-2 (0.97). The improvement in $I_{\mathrm{D}} / I_{\mathrm{G}}$ of $\mathrm{BC} / \mathrm{GO}-2$ over $\mathrm{GO}$ can be ascribed to the removal of some oxygen-containing functional groups on the surface of GO [40], since GO can be deoxygenated in alkaline solutions [41] during sample preparation (boiled with a $0.5-\mathrm{M} \mathrm{NaOH}$ solution for $15 \mathrm{~min}$ and cleaned with 1 wt\% $\mathrm{NaOH}$ for 2 days), in line with our previous work [21]. As shown in Fig. 3d, besides the D and G bands, two peaks located at 2680 and $2931 \mathrm{~cm}^{-1}$ are also noted in the spectra of GO and $\mathrm{BC} / \mathrm{GO}-2$, which can be assigned to the 


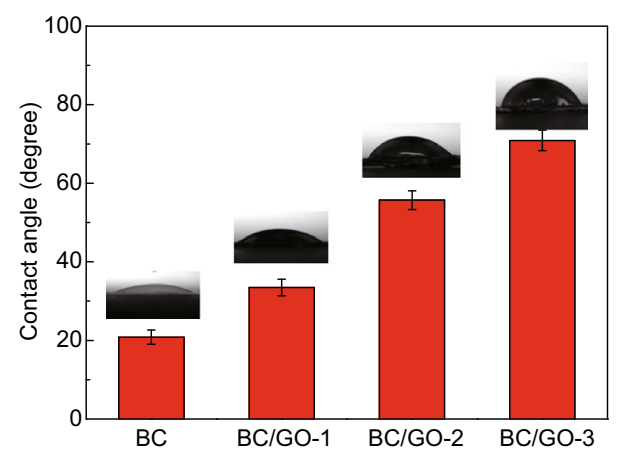

Fig. 5 Water contact angles of $\mathrm{BC}$ and $\mathrm{BC} / \mathrm{GO}$ nanocomposites

$2 \mathrm{D}$ and $\mathrm{D}+\mathrm{G}$ bands, respectively [42]. The $2 \mathrm{D}$ band is highly sensitive to the stacking of graphene, and the intensity ratio of $2 \mathrm{D}$ band to $\mathrm{G}$ band $\left(I_{2 \mathrm{D}} / I_{\mathrm{G}}\right)$ is an important parameter to evaluate the layering of graphene materials $[42,43]$. The $I_{2 \mathrm{D}} / I_{\mathrm{G}}$ ratios of single-, double-, triple-, and multi- $(>4)$ layer sheets are typically $>1.6, \sim 0.8, \sim 0.30$, and $\sim 0.07$, respectively [43]. The calculated $I_{2 \mathrm{D}} / I_{\mathrm{G}}$ is 0.66 and 0.68 for $\mathrm{GO}$ and $\mathrm{BC} /$ GO-2, respectively, which suggests a few-layered texture of $\mathrm{GO}$ in $\mathrm{BC} / \mathrm{GO}-2$, in accordance with the specification of the providers of the $\mathrm{GO}$ suspension.

The pore structures of the $\mathrm{BC} / \mathrm{GO}$ nanocomposites and pure $\mathrm{BC}$ (in aerogel) were analyzed by $\mathrm{N}_{2}$ adsorptiondesorption measurements (Fig. 4). Note that both BC/GO and $\mathrm{BC}$ exhibit a characteristic type IV isotherm, suggesting the presence of many mesopores in each material, which can be further evidenced by the pore size distribution shown in the insets of Fig. 4a-d. The BET specific surface areas of $\mathrm{BC}, \mathrm{BC} / \mathrm{GO}-1, \mathrm{BC} / \mathrm{GO}-2$, and $\mathrm{BC} /$ GO-3 are 156.6, 157.9, 163.6, and $165.4 \mathrm{~m}^{2} \mathrm{~g}^{-1}$, respectively. These results indicate that the incorporation of GO does not significantly affect the BET specific surface area of BC.

Surface hydrophilicity of the $\mathrm{BC} / \mathrm{GO}$ nanocomposites was assessed by measuring their water contact angles (Fig. 5). As expected, the water contact angles of the $\mathrm{BC} /$ GO nanocomposites show an increasing trend with the GO content of the $\mathrm{BC} / \mathrm{GO}$ nanocomposites. This indicates that the incorporation of $\mathrm{GO}$ into $\mathrm{BC}$ decreases the hydrophilicity of the BC/GO nanocomposites. However, all BC/GO nanocomposites demonstrate favorable hydrophilicity (water contact angles $<90^{\circ}$ ).

Mechanical properties of $\mathrm{BC}$ and $\mathrm{BC} / \mathrm{GO}$ hydrogels were tested under tensile loads (Fig. 6). The typical tensile stress-strain curves shown in Fig. 6a reveal an increasing trend in the peak load and decreasing trend in strain at break with the increase in GO content. Data presented in Fig. $6 \mathrm{~b}, \mathrm{c}$ demonstrate that the tensile strength and modulus of $\mathrm{BC} / \mathrm{GO}$ nanocomposite hydrogels are greatly improved over those of bare $\mathrm{BC}$, and the improvements are dependent on the GO content. Figure 6d shows the opposite trend in strain at break with the GO content as compared to the tensile strength and modulus, as expected.
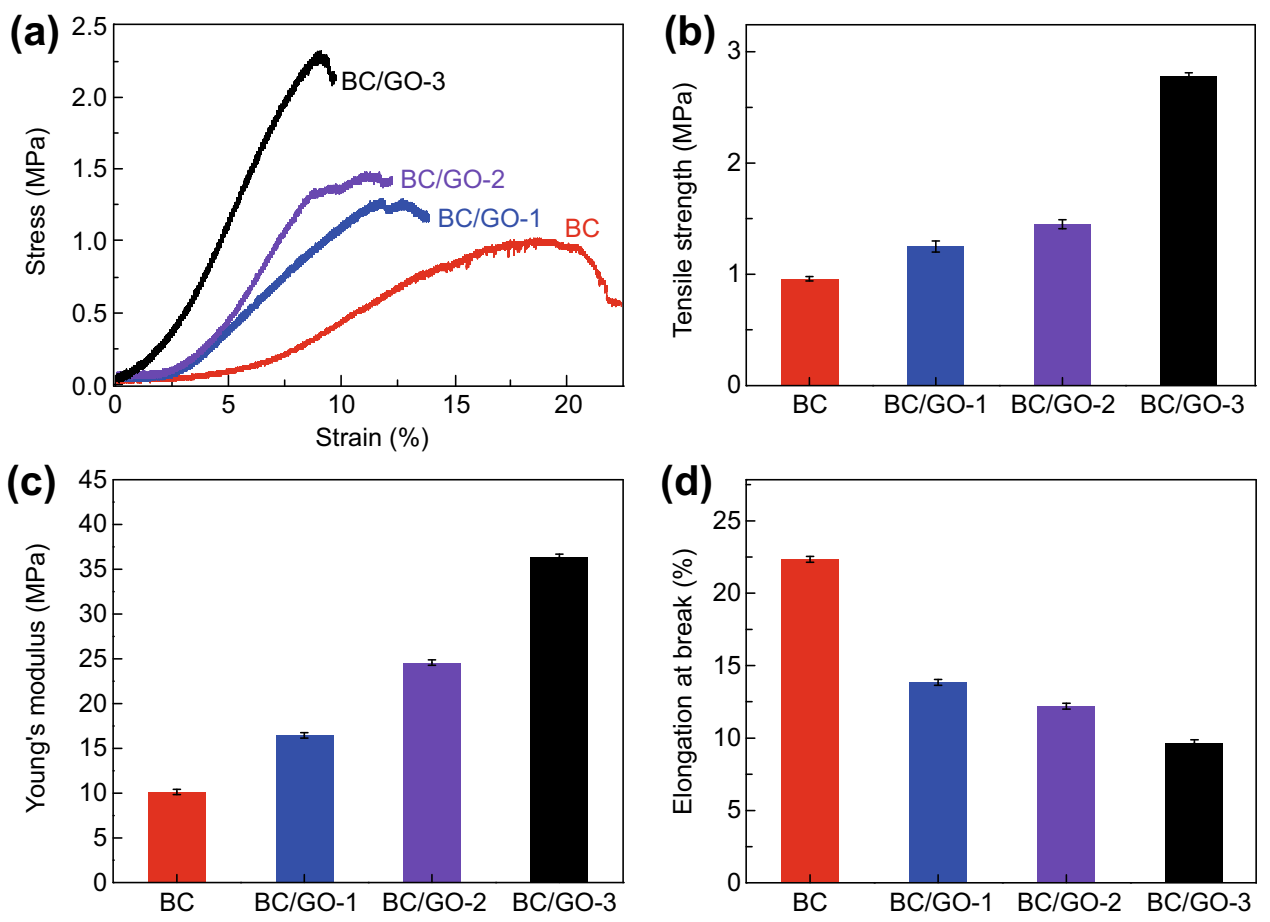

Fig. 6 Typical a stress-strain, $\mathbf{b}$ tensile strength, $\mathbf{c}$ tensile modulus, and $\mathbf{d}$ strain at break of BC and BC/GO nanocomposites 


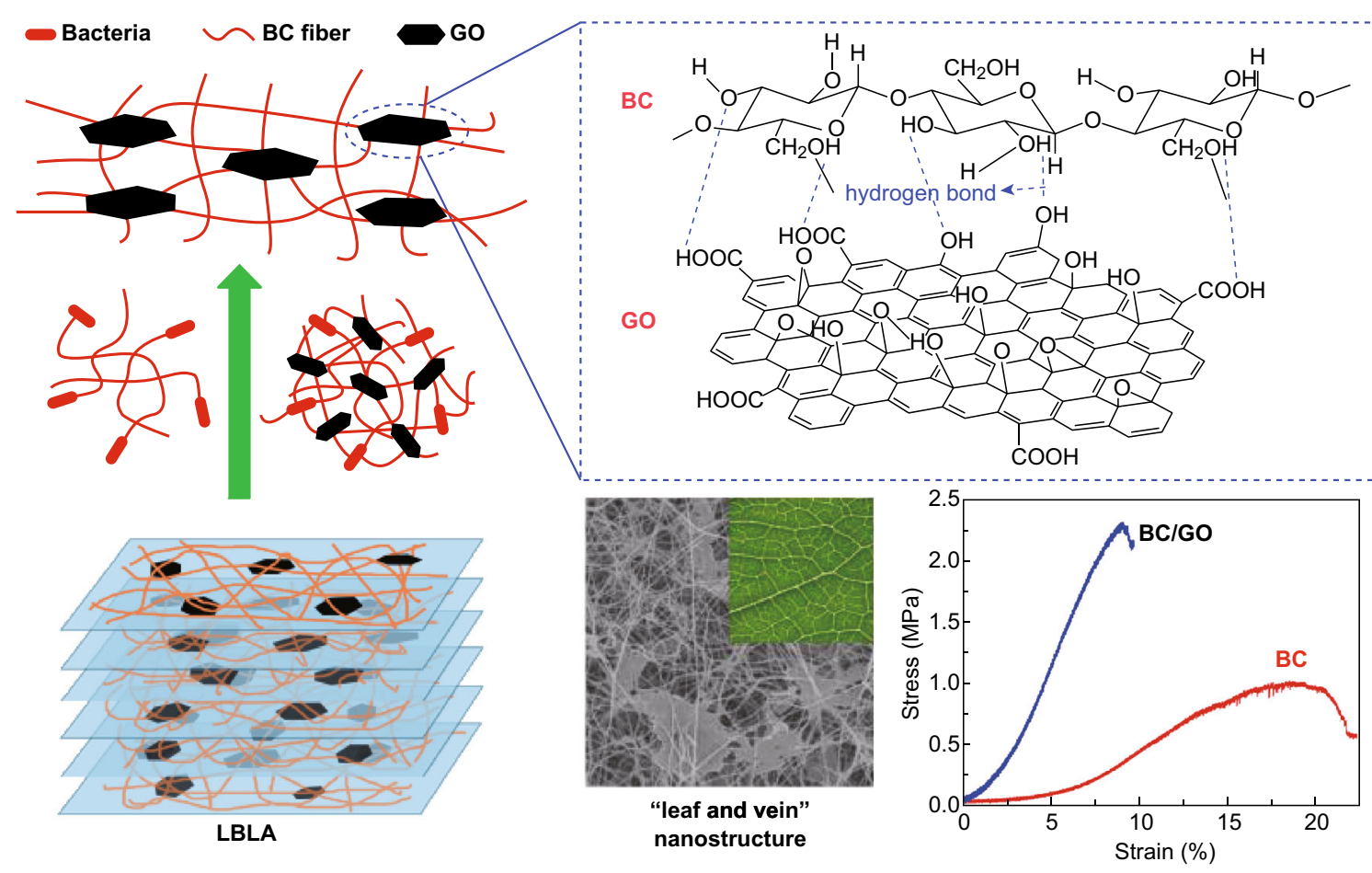

Fig. 7 Schematic diagram of "leaf and vein" nanostructure of BC/GO prepared by LBLA strategy. The layer-by-layer culture mode facilitates the bundling of 2D GO nanosheets by 1D BC nanofibers and promotes distribution of 2D GO nanosheets in 3D BC matrix

Among the three $\mathrm{BC} / \mathrm{GO}$ samples, BC/GO-3 has the highest tensile strength $(2.78 \pm 0.03 \mathrm{MPa})$ and modulus $(36.4 \pm 0.3 \mathrm{MPa})$, which are 2.9 and 3.6 times those of bare $\mathrm{BC}$, respectively. These improvements are much higher than the increases in $\mathrm{BC} / 0.19 \mathrm{wt} \% \mathrm{GO}(3.7 \%$ and $32.2 \%$, respectively) and $\mathrm{BC} / 0.29 \mathrm{wt} \% \mathrm{GO}(21.4 \%$ and $62.7 \%$, respectively) prepared by conventional static culture [21]. Furthermore, the improvements are also higher than other reported data. For instance, Liang et al. [44] observed a $76 \%$ increase in tensile strength and a $62 \%$ improvement in Young's modulus when $0.7 \mathrm{wt} \% \mathrm{GO}$ was added in a poly(vinyl alcohol) matrix. Zhang et al. [45] claimed a $132 \%$ increase in tensile strength and a $36 \%$ improvement in compressive strength when $0.8 \mathrm{wt} \% \mathrm{GO}$ was incorporated in a poly(vinyl alcohol) hydrogel.

Such immense improvements in LBLA-derived BC/GO hydrogels can be explained by the following factors (Fig. 7). Firstly, the formation of hydrogen bonding between $\mathrm{BC}$ and GO, as revealed by FTIR shown in Fig. $3 b$, ensures tight bonding between the 1D and 2D components. Secondly, the layer-by-layer culture mode improves the dispersion of 2D GO nanosheets in the 3D BC matrix. Finally, the layer-by-layer culture mode facilitates the mechanical bundling of 2D GO nanosheets by the 1D $\mathrm{BC}$ nanofibers, which results in a leaf/vein-like structure. Strong hydrogen bonding, close mechanical bundling, and even distribution are responsible for the tremendous improvements in mechanical properties.

\section{Conclusions}

BC/GO nanocomposites with a sophisticated nanostructure have been fabricated via a novel in situ LBLA strategy. The LBLA method involves two simultaneous steps: selfassembly of 1D BC nanofibers into a 3D structure and selfbundling of 2D GO nanosheets by $1 \mathrm{D}$ BC nanofibers. The $\mathrm{BC} / \mathrm{GO}$ hydrogels show greatly improved mechanical properties over those of bare $\mathrm{BC}$ and other $\mathrm{BC} / \mathrm{GO}$ counterparts prepared by conventional static culture methods. The intriguing nanostructure with strong hydrogen bonding, close mechanical bundling, and even distribution of 2D GO nanosheets throughout the 3D BC network are the main reasons why the LBLA-derived BC/GO hydrogels are ultra-strong. These results show that such a novel LBLA strategy has great promise in the development of highperformance BC-based hydrogels for various applications.

Acknowledgements This work is supported by the National Natural Science Foundation of China (Grant Nos. 51572187, 51563008, 51662009, 31660264), the Provincial Natural Science Foundation of Jiangxi (Grant No. 20161BAB206149), and the Key Project of Natural Science Foundation of Jiangxi Province (Grant No. 20161ACB20018).

Open Access This article is distributed under the terms of the Creative Commons Attribution 4.0 International License (http://creativecommons. org/licenses/by/4.0/), which permits unrestricted use, distribution, and reproduction in any medium, provided you give appropriate credit to the original author(s) and the source, provide a link to the Creative Commons license, and indicate if changes were made. 


\section{References}

1. N. Dubey, R. Bentini, I. Islam, T. Cao, A.H.C. Neto, V. Rosa, Graphene: a versatile carbon-based material for bone tissue engineering. Stem Cells Int. 2015, 804213 (2015). https://doi.org/ $10.1155 / 2015 / 804213$

2. S. Goenka, V. Sant, S. Sant, Graphene-based nanomaterials for drug delivery and tissue engineering. J. Control. Release 173, 75-88 (2014). https://doi.org/10.1016/j.jconrel.2013.10.017

3. M. Gu, Y.S. Liu, T. Chen, F. Du, X.H. Zhao, C.Y. Xiong, Y.S. Zhou, Is graphene a promising nano-material for promoting surface modification of implants or scaffold materials in bone tissue engineering? Tissue Eng. Part B 20(5), 477-491 (2014). https://doi.org/10.1089/ten.teb.2013.0638

4. G. Lalwani, A. Gopalan, M. D'Agati, J.S. Sankaran, S. Judex, Y.X. Qin, B. Sitharaman, Porous three-dimensional carbon nanotube scaffolds for tissue engineering. J. Biomed. Mater. Res. Part A 103(10), 3212-3225 (2015). https://doi.org/10.1002/jbm.a. 35449

5. P. Newman, Z. Lu, S.I. Roohani-Esfahani, T.L. Church, M. Biro et al., Porous and strong three-dimensional carbon nanotube coated ceramic scaffolds for tissue engineering. J. Mater. Chem. B 3(42), 8337-8347 (2015). https://doi.org/10.1039/c5tb01052g

6. D.A. Stout, Recent advancements in carbon nanofiber and carbon nanotube applications in drug delivery and tissue engineering. Curr. Pharm. Des. 21(15), 2037-2044 (2015). https://doi.org/10. 2174/1381612821666150302153406

7. O. Akhavan, Graphene scaffolds in progressive nanotechnology/ stem cell-based tissue engineering of the nervous system. J. Mater. Chem. B 4(19), 3169-3190 (2016). https://doi.org/10. 1039/c6tb00152a

8. H. Wen, C. Yin, A. Du, L. Deng, Y. He, L. He, Folate conjugated PEG-chitosan/graphene oxide nanocomplexes as potential carriers for pH-triggered drug release. J. Control. Release 213, e44e45 (2015). https://doi.org/10.1016/j.jconrel.2015.05.072

9. H. Yang, D.H. Bremner, L. Tao, H. Li, J. Hu, L. Zhu, Carboxymethyl chitosan-mediated synthesis of hyaluronic acid-targeted graphene oxide for cancer drug delivery. Carbohydr. Polym. 135, 72-78 (2016). https://doi.org/10.1016/j.carbpol. 2015.08.058

10. T.H. Tran, H.T. Nguyen, T.T. Pham, J.Y. Choi, H.-G. Choi, C.S. Yong, J.O. Kim, Development of a graphene oxide nanocarrier for dual-drug chemo-phototherapy to overcome drug resistance in cancer. ACS Appl. Mater. Interfaces 7(51), 28647-28655 (2015). https://doi.org/10.1021/acsami.5b10426

11. Y. Jin, J. Wang, H. Ke, S. Wang, Z. Dai, Graphene oxide modified PLA microcapsules containing gold nanoparticles for ultrasonic/CT bimodal imaging guided photothermal tumor therapy. Biomaterials 34(20), 4794-4802 (2013). https://doi.org/ 10.1016/j.biomaterials.2013.03.027

12. C. Chung, Y.-K. Kim, D. Shin, S.-R. Ryoo, B.H. Hong, D.-H. Min, Biomedical applications of graphene and graphene oxide. Acc. Chem. Res. 46(10), 2211-2224 (2013). https://doi.org/10. 1021/ar300159f

13. H. Bai, C. Li, G. Shi, Functional composite materials based on chemically converted graphene. Adv. Mater. 23(9), 1089-1115 (2011). https://doi.org/10.1002/adma.201003753

14. X. Shi, H. Chang, S. Chen, C. Lai, A. Khademhosseini, H. Wu, regulating cellular behavior on few-layer reduced graphene oxide films with well-controlled reduction states. Adv. Funct. Mater. 22(4), 751-759 (2012). https://doi.org/10.1002/adfm.201102305

15. Y. Zhang, T.R. Nayak, H. Hong, W. Cai, Graphene: a versatile nanoplatform for biomedical applications. Nanoscale 4(13), 3833-3842 (2012). https://doi.org/10.1039/C2NR31040F
16. X.T. Zheng, X.Q. Ma, C.M. Li, Highly efficient nuclear delivery of anti-cancer drugs using a bio-functionalized reduced graphene oxide. J. Colloid Interface Sci. 467, 35-42 (2016). https://doi.org/ 10.1016/j.jcis.2015.12.052

17. Y. Feng, X. Zhang, Y. Shen, K. Yoshino, W. Feng, A mechanically strong, flexible and conductive film based on bacterial cellulose/graphene nanocomposite. Carbohydr. Polym. 87(1), 644-649 (2012). https://doi.org/10.1016/j.carbpol.2011.08.039

18. Y. Liu, J. Zhou, E. Zhu, J. Tang, X. Liu, W. Tang, Facile synthesis of bacterial cellulose fibres covalently intercalated with graphene oxide by one-step cross-linking for robust supercapacitors. J. Mater. Chem. C 3(5), 1011-1017 (2015). https://doi.org/ 10.1039/C4TC01822B

19. C. Gao, Y. Wan, C. Yang, K. Dai, T. Tang, H. Luo, J. Wang, Preparation and characterization of bacterial cellulose sponge with hierarchical pore structure as tissue engineering scaffold. J. Porous Mater. 18(2), 139-145 (2011). https://doi.org/10.1007/ s10934-010-9364-6

20. S.H. Yoon, H.J. Jin, M.C. Kook, Y.R. Pyun, Electrically conductive bacterial cellulose by incorporation of carbon nanotubes. Biomacromolecules 7(4), 1280-1284 (2006). https://doi.org/10. 1021/bm050597g

21. H. Si, H. Luo, G. Xiong, Z. Yang, S.R. Raman, R. Guo, Y. Wan, One-step in situ biosynthesis of graphene oxide-bacterial cellulose nanocomposite hydrogels. Macromol. Rapid Commun. 35(19), 1706-1711 (2014). https://doi.org/10.1002/marc. 201400239

22. Y.Z. Wan, Y. Huang, C.D. Yuan, S. Raman, Y. Zhu, H.J. Jiang, F. He, C. Gao, Biomimetic synthesis of hydroxyapatite/bacterial cellulose nanocomposites for biomedical applications. Mater. Sci. Eng. C 27(4), 855-864 (2007). https://doi.org/10.1016/j.msec. 2006.10.002

23. L. Hong, Y.L. Wang, S.R. Jia, Y. Huang, C. Gao, Y.Z. Wan, Hydroxyapatite/bacterial cellulose composites synthesized via a biomimetic route. Mater. Lett. 60(13-14), 1710-1713 (2006). https://doi.org/10.1016/j.matlet.2005.12.004

24. L. Segal, J.J. Creely, A.E. Martin, C.M. Conrad, An empirical method for estimating the degree of crystallinity of native cellulose using the X-ray diffractometer. Text. Res. J. 29(10), 786-794 (1959). https://doi.org/10.1177/004051755902901003

25. H. Luo, G. Xiong, Z. Yang, S.R. Raman, H. Si, Y. Wan, A novel three-dimensional graphene/bacterial cellulose nanocomposite prepared by in situ biosynthesis. RSC Adv. 4(28), 14369-14372 (2014). https://doi.org/10.1039/C4RA00318G

26. S. Taokaew, S. Seetabhawang, P. Siripong, M. Phisalaphong, Biosynthesis and characterization of nanocellulose-gelatin films. Materials 6(3), 782-794 (2013). https://doi.org/10.3390/ ma6030782

27. C.H. Haigler, A.R. White Jr., K.M. Cooper, Alteration of in vivo cellulose ribbon assembly by carboxymethylcellulose and other cellulose derivatives. J. Cell Biol. 94(1), 64-69 (1982). https:// doi.org/10.1083/jcb.94.1.64

28. H.-P. Cong, P. Wang, S.-H. Yu, Stretchable and self-healing graphene oxide-polymer composite hydrogels: a dual-network design. Chem. Mater. 25(16), 3357-3362 (2013). https://doi.org/ $10.1021 / \mathrm{cm} 401919 \mathrm{c}$

29. W. Ouyang, J. Sun, J. Memon, C. Wang, J. Geng, Y. Huang, Scalable preparation of three-dimensional porous structures of reduced graphene oxide/cellulose composites and their application in supercapacitors. Carbon 62, 501-509 (2013). https://doi. org/10.1016/j.carbon.2013.06.049

30. W. Shao, H. Liu, X. Liu, S. Wang, R. Zhang, Anti-bacterial performances and biocompatibility of bacterial cellulose/graphene oxide composites. RSC Adv. 5(7), 4795-4803 (2014). https://doi.org/10.1039/C4RA13057J 
31. Y. Xu, W. Hong, H. Bai, C. Li, G. Shi, Strong and ductile poly(vinyl alcohol)/graphene oxide composite films with a layered structure. Carbon 47(15), 3538-3543 (2009). https://doi.org/ 10.1016/j.carbon.2009.08.022

32. R. Liu, L. Ma, S. Huang, J. Mei, J. Xu, G. Yuan, Large areal mass, flexible and freestanding polyaniline/bacterial cellulose/graphene film for high-performance supercapacitors. RSC Adv. 6(109), 107426-107432 (2016). https://doi.org/10.1039/ C6RA21920A

33. H. Luo, J. Dong, Y. Zhang, G. Li, R. Guo et al., Constructing 3D bacterial cellulose/graphene/polyaniline nanocomposites by novel layer-by-layer in situ culture toward mechanically robust and highly flexible freestanding electrodes for supercapacitors. Chem. Eng. J. 334, 1148-1158 (2018). https://doi.org/10.1016/j. cej.2017.11.065

34. N.F. Vasconcelos, J.P. Feitosa, G.F. Da, J.P. Morais, F.K. Andrade, D.S.F. Ms, M.F. Rosa, Bacterial cellulose nanocrystals produced under different hydrolysis conditions: properties and morphological features. Carbohydr. Polym. 155, 425-431 (2017). https://doi.org/10.1016/j.carbpol.2016.08.090

35. S. Park, K.S. Lee, G. Bozoklu, W. Cai, S.B.T. Nguyen, R.S. Ruoff, Graphene oxide papers modified by divalent ions-enhancing mechanical properties via chemical cross-linking. ACS Nano 2(3), 572-578 (2008). https://doi.org/10.1021/nn700349a

36. N. Song, D. Jiao, P. Ding, S. Cui, S. Tang, L.Y. Shi, Anisotropic thermally conductive flexible films based on nanofibrillated cellulose and aligned graphene nanosheets. J. Mater. Chem. C 4(2), 305-314 (2015). https://doi.org/10.1039/C5TC02194D

37. N. Song, X. Hou, L. Chen, S. Cui, L. Shi, P. Ding, A green plastic constructed from cellulose and functionalized graphene with high thermal conductivity. ACS Appl. Mater. Interfaces 9(21), 17914-17922 (2017). https://doi.org/10.1021/acsami.7b02675

38. D. Yang, A. Velamakanni, G. Bozoklu, S. Park, M. Stoller et al., Chemical analysis of graphene oxide films after heat and chemical treatments by X-ray photoelectron and micro-Raman spectroscopy. Carbon 47(1), 145-152 (2009). https://doi.org/10. 1016/j.carbon.2008.09.045

39. N.D. Luong, N. Pahimanolis, U. Hippi, J.T. Korhonen, J. Ruokolainen, L.-S. Johansson, J.-D. Nam, J. Seppälä, Graphene/cellulose nanocomposite paper with high electrical and mechanical performances. J. Mater. Chem. 21(36), 13991-13998 (2011). https://doi.org/10.1039/C1JM12134K

40. Z. Fan, K. Wang, T. Wei, J. Yan, L. Song, B. Shao, An environmentally friendly and efficient route for the reduction of graphene oxide by aluminum powder. Carbon 48(5), 1686-1689 (2010). https://doi.org/10.1016/j.carbon.2009.12.063

41. X. Fan, W. Peng, Y. Li, X. Li, S. Wang, G. Zhang, F. Zhang, Deoxygenation of exfoliated graphite oxide under alkaline conditions: a green route to graphene preparation. Adv. Mater. 20(23), 4490-4493 (2008). https://doi.org/10.1002/adma. 200801306

42. K. Krishnamoorthy, M. Veerapandian, K. Yun, S.J. Kim, The chemical and structural analysis of graphene oxide with different degrees of oxidation. Carbon 53, 38-49 (2013). https://doi.org/10. 1016/j.carbon.2012.10.013

43. O. Akhavan, Bacteriorhodopsin as a superior substitute for hydrazine in chemical reduction of single-layer graphene oxide sheets. Carbon 81(1), 158-166 (2015). https://doi.org/10.1016/j. carbon.2014.09.044

44. J. Liang, Y. Huang, L. Zhang, Y. Wang, Y. Ma, T. Guo, Y. Chen, Molecular-level dispersion of graphene into poly(vinyl alcohol) and effective reinforcement of their nanocomposites. Adv. Funct. Mater. 19(14), 2297-2302 (2009). https://doi.org/10.1002/adfm. 200801776

45. L. Zhang, Z. Wang, C. Xu, Y. Li, J. Gao, W. Wang, Y. Liu, High strength graphene oxide/polyvinyl alcohol composite hydrogels. J. Mater. Chem. 21(28), 10399-10406 (2011). https://doi.org/10. 1039/COJM04043F 